\title{
Terapia sexual y normalización: significados del malestar sexual en mujeres y hombres diagnosticados con disfunción
} sexual $^{*}$

| 'Eva Alcántara Zavala, ${ }^{2}$ Ana Amuchástegui Herrera |

Resumen: En los últimos veinticinco años, instituciones y profesionales interesados en brindar terapia sexual se han establecido en las áreas urbanas de México. El tratamiento de las disfunciones sexuales es tanto farmacéutico como conductual. Estudios sociohistóricos han señalado las particularidades y limitaciones de las aproximaciones biomédicas a los malestares sexuales. En este estudio presentamos una serie de reflexiones en torno a la noción de disfunción sexual y los presupuestos bioconductuales que sostienen su tratamiento en terapia sexual. El objetivo del estudio fue comprender los significados del malestar sexual en un grupo abierto y mixto de terapia sexual, que se llevó a cabo en la ciudad de México en sesiones semanales de dos horas. Aunque se realizaron observaciones de las sesiones durante un año, para un análisis detallado se seleccionaron veinte de ellas. En esas sesiones participaron intermitentemente tres mujeres terapeutas sexuales, y quince mujeres y diez hombres diagnosticados con algún tipo de disfunción sexual. Un análisis cualitativo de los diálogos demostró que los significados de malestar sexual están fuertemente ligados al establecimiento de un modelo ideal de normalidad sexual. Este modelo se refiere a un estándar de desempeño sexual al cual las personas debían adaptarse con el objetivo de cumplir con los estándares de normalidad. El género es un elemento constitutivo de dicho modelo, inequidades y relaciones significantes de poder son reguladas, reproducidas y reafirmadas a través del modelo que sostiene la terapia sexual.

> Palabras clave: heterosexualidad; terapia sexual; disfunción sexual; sexología.
1 Profesora-investigadora del
Departamento de Educación y
Comunicación de la
Universidad Autónoma
Metropolitana-Xochimilco.
Maestra en Estudios de la
Mujer y candidata a Doctora en
Ciencias Sociales por la misma
universidad. Correo
electrónico:
eazavala@correo.xoc.uam.mx.
2Profesora-investigadora del
Departamento de Educación y
Comunicación de la
Universidad Autónoma
Metropolitana-Xochimilco
Doctora en Filosofía por la
Universidad de Londres. Correo
electrónico:
aamuchastegui@prodigy.net.mx. 


\section{Introducción}

En México, al igual que en otros países, el malestar sexual ha sido abordado principalmente desde el enfoque sexológico en torno a la noción de disfunción sexual. Sin embargo, diversos estudios enmarcados en el feminismo y en el enfoque sociohistórico de la sexualidad han cuestionado tanto a la sexología como su conceptualización del malestar sexual. Esos estudios coinciden en señalar que la aproximación de la sexología es esencialista, es decir, que esta scientia sexualis (FOUCAULT, 2000) intenta explicar un proceso complejo y múltiple, como es el ejercicio del placer, basándose en una supuesta esencia o verdad interna, lo cual supone una sexualidad conformada a partir de esquemas únicos, básicos y uniformes (WEEKS, 2000). También se reitera que la sexología ha sustentado, legitimizado y universalizado un modelo de sexualidad basado en criterios androcéntricos (JACKSON, 1987; KOEDT, 2001; GONEMAN, 1994). El presente estudio muestra la relevancia de las críticas dirigidas a la conceptualización sexológica de la sexualidad al enfocar su atención en un tema que ha cobrado un creciente y vertiginoso interés en la actualidad: las disfunciones sexuales y su tratamiento mediante diversas técnicas que conforman la terapia sexual. Para ello, resulta fundamental comprender la construcción histórica de la noción de disfunción sexual y el establecimiento de la terapia sexual como una práctica legitimizada a partir de su encadenamiento con la medicina y la biología.

\section{Breves notas acerca de la normalidad sexual}

Foucault (2000) refiere que a partir del siglo XIX se desarrolla una creciente preocupación por los estudios científicos hacia diversos aspectos considerados como sexuales, la cual desemboca en la construcción de la sexualidad como objeto de la ciencia. Desde su inicio, esta ciencia sexual se conforma como una relación de poder que mediante técnicas establecidas intenta producir discursos verdaderos sobre el sexo, avalados por la institución médica que en forma implícita lleva la exigencia de normalidad. Según Weeks (1993), los sexólogos han jugado un importante papel para definir, inventariar, describir y analizar las manifestaciones concebidas como patológicas en la vida sexual. Así, la sexología se ha construido mediante un esfuerzo sostenido para darle al sexo y sus 
manifestaciones un carácter científico, mediante el aislamiento y la individualización de supuestas características específicas de la sexualidad, detallando los caminos de la normalidad y sus variaciones anormales.

Si tomamos en cuenta la investigación sociohistórica, encontramos que el concepto de normalidad sexual se ha conformado y transformado a través del tiempo a partir de diversos discursos y en un contexto de relaciones de poder. La definición de anormalidad ha surgido generalmente de una variedad de prácticas sexuales ajenas al coito reproductivo, lo que apoya la existencia de una supuesta heterosexualidad natural.

Investigaciones como la de Kinsey y Masters y Johnson resultaron fundamentales en la construcción de la ciencia sexual y la delimitación de los criterios de normalidad sexual. Por ejemplo, el ciclo de la respuesta sexual humana (MASTERS; JOHNSON,1976a) fue construido a partir del registro de los cambios anatomofisiológicos que experimenta el cuerpo humano en el transcurso de una relación coital vaginal y con el tiempo ha llegado a representar la prueba científica que avala la existencia de un patrón único que permite el acceso al placer sexual. Es decir, el modelo caracterizado por las cuatro fases que comprenden un ciclo de excitación, meseta, orgasmo y resolución, se presenta como la contraparte objetiva de la subjetiva sensación de placer sexual. Entonces, se establece que es indispensable cubrir dicho ciclo para experimentar placer sexual y se diseñan una serie de tecnologías para regular la respuesta sexual y adecuarla a los patrones preestablecidos. Así surge la terapia sexual como un tratamiento para que las parejas adapten su respuesta sexual al ciclo, la cual fue propuesta en primera instancia por Masters y Johnson y desarrollada a la par de sus investigaciones sobre la respuesta sexual humana.

Otra autora importante para el desarrollo de la terapia sexual ha sido Kaplan (1978a; 1985) quien reformula las fases del modelo de respuesta sexual al incorporar la fase de deseo. Ella propone un modelo de respuesta sexual que incluye tres fases: deseo, exitación y orgasmo. Para Kaplan, la etiología de las disfunciones sexuales se divide en profunda (cuando la disfunción se relaciona con factores insertados en la estructura de la personalidad) e inmediata (cuando tiene que ver con factores presentes en el aquí y el ahora). También reformula el tratamiento al recomendar que un solo terapeuta trabaje con ambos miembros de la pareja.

Desde un inicio, las tareas sexuales se establecieron como el centro alrededor del cual giraba el tratamiento en la terapia sexual. Masters y Jonhson (1976a) 
diseñaron un programa terapéutico de pasos progresivos con base en la asignación de tareas, cuyo objetivo era adaptar la respuesta sexual de las parejas a los patrones establecidos como normales. Por su parte, Kaplan (1978a) utiliza el término "tareas sexuales” para denominar la prescripción de experiencias eróticas específicas y afirma que dichas prescripciones son el rasgo distintivo de la terapia sexual. Estas tareas incluyen, entre otras, observar en un espejo el cuerpo desnudo, actividades de comunicación e intercambio con la pareja, entrenamiento en la masturbación y ejercicios durante el coito (MASTERS; JOHNSON, 1976b; KAPLAN, 1978b; ÁLVAREZ-GAYOU, 1986). Las tareas sexuales que se manejaban en el grupo de terapia sexual observado son muy similares a las descritas por esos autores.

En la actualidad, las disfunciones sexuales han adquirido la categoría de entidades clínicas y se encuentran descritas dentro del capítulo $\mathrm{V}$ de la Clasificación Internacional de Enfermedades en su décima revisión (CIE-10, 1995) editado por la Organización Mundial de la Salud, así como en el Manual de Diagnóstico y Estadística de la Asociación Psiquiátrica Americana (DSM IV, 1995). En la sección de Trastornos sexuales y de la identidad sexual del DSM IV, se definen las disfunciones sexuales como "una alteración del deseo sexual, por cambios psicofisiológicos en el ciclo de la respuesta sexual y por la provocación de malestar y problemas interpersonales" (DSM IV, 1995:505). El DSM IV incluye seis diferentes grupos de trastornos sexuales y cada grupo clasifica trastornos específicos; para precisar el diagnóstico, se deben señalar algunos detalles que incluyen si el trastorno es de toda la vida o adquirido, general o situacional, debido a factores psicológicos o combinados. Para definir y clasificar las disfunciones sexuales, ambos compendios parten del modelo de respuesta sexual humana de Masters y Johnson (1976a) y su clasificación incluye algunas consideraciones sobre la fase de deseo teorizadas por Kaplan (1985).

Así, la visión de las disfunciones sexuales guarda estrecha relación con la respuesta sexual considerada normal, de manera que mientras la variación se aleje más de dicho patrón, aumentará la posibilidad de considerarla como disfuncional. Sin embargo, es oportuno recordar que la respuesta sexual ha sido construida a partir de concepciones androcéntricas de sexualidad. Un ejemplo de ello es que la forma más frecuente del trastorno orgásmico masculino es cuando "el varón no consigue llegar al orgasmo durante el coito, pero puede eyacular con otros tipos de estimulación, por ejemplo, manual u oral" (DSM IV, 1995, p. 520). Como en 
este caso, las descripciones de la disfunción privilegian la penetración y el coito como las actividades genuinas y más gratificantes para hombres y mujeres.

Para autoras como Tiefer (1995), la relevancia de analizar las nociones contenidas en el DSM IV estriba en la importancia que actualmente tiene dicho texto, el cual es utilizado en distintos países como una referencia autorizada en tribunales, hospitales, investigaciones y escuelas. Compartimos con Tiefer la idea de que:

La clasificación de la conducta humana brinda terreno fértil al control social tanto en el nivel social como en el imaginativo. La clasificación supone crear diferencias que, en nuestra cultura, implican inexorablemente una desigualdad y una jerarquía. En consecuencia, pone en marcha unos procesos en los que a menudo se incluye la intimidación y estigmatización de ciertos grupos, estableciendo y exigiendo la aplicación de normas, la creación de un lenguaje y unas imágenes culturalmente dominantes y, no en menor medida, la constitución y conformación de deseos y necesidades individuales, como los revolucionarios han señalado repetidas veces designar es poder (TIEFER, 1995 p. 166 - cursivas en el original).

\section{El escenario de estudio}

La primera autora realizó observaciones en un grupo de terapia sexual por periodo de un año. Durante ese tiempo, el grupo trabajaba una vez por semana por un lapso de dos horas. En el momento en que se realizó el estudio, el grupo se definía como abierto y mixto, esto es, las personas denominadas pacientes eran hombres y mujeres que se reunían en el mismo espacio e iniciaban y concluían su estancia en el grupo intermitentemente.

En la mayoría de las sesiones, el trabajo del grupo se dividía en tres partes. Durante la primera y por un periodo aproximado de veinte minutos, el grupo trabajaba con movimientos corporales y de relajación. En un segundo momento, el grupo se sentaba en círculo y alguna de las terapeutas hacía preguntas generales acerca de la actividad anterior. También eran tratados temas de interés grupal, como pagos, presentación de integrantes, despedidas, desacuerdos o reglas; ocasionalmente el grupo trabajó en esta modalidad hasta el final de la sesión. En la parte final y con frecuencia la que abarcaba el mayor tiempo de la sesión, el grupo se dividía en subgrupos - según el número de terapeutas, habitualmente tres - que dialogaban principalmente en torno a las denominadas tareas que les eran encomendadas en sesiones anteriores. ${ }^{1}$ 
Las tareas sexuales se referían a una serie de actividades que las personas debían realizar en su casa y reportar durante las sesiones de terapia; a decir de las terapeutas, esas tareas sexuales se indicaban principalmente utilizando el esquema de Kaplan, aunque realizaban algunas adaptaciones. Las tareas eran asignadas por las terapeutas en el inicio del tratamiento con base en dos listas preexistentes y constituían una importante guía en el trabajo del grupo. Aunque las listas de actividades eran diferentes para hombres y para mujeres, algunas se encontraban en ambas. Dichas actividades tenían una secuencia, en donde para avanzar a la siguiente, las personas debían reportar en la sesión el ejercicio. Entonces la terapeuta evaluaba si era tiempo de asignar una nueva actividad o si se debía continuar con la misma. En ocasiones, las y los pacientes reportaban no haber hecho la tarea y hablaban al respecto.

\section{Participantes}

El grupo estaba conformado por tres psicoterapeutas, todas psicólogas con estudios especializados en sexología y algunas con formación en psicoanálisis o terapia familiar. Durante el lapso de observación del grupo, sus participantes variaron debido a que como hemos comentado, era un grupo abierto. Durante el año de observación, la asistencia osciló entre seis y dieciséis pacientes por sesión. Las personas que asistían habían sido diagnosticadas con disfunción sexual en una primera entrevista individual llevada a cabo por alguna de las terapeutas. Quince mujeres y diez hombres participaron en las sesiones seleccionadas para el análisis. Los cuadros 1 y 2 especifican algunos de sus datos. 
Cuadro 1 - Algunos datos de mujeres que participaron en las sesiones seleccionadas para el análisis

\begin{tabular}{|c|c|c|c|c|c|c|}
\hline \multicolumn{7}{|c|}{ MUJERES } \\
\hline Paciente & Edad & Escolaridad & $\begin{array}{c}\text { Estado } \\
\text { civil }\end{array}$ & Ocupación & $\begin{array}{l}\text { Lugar de } \\
\text { residencia }\end{array}$ & Referencia \\
\hline PM1 & 29 & licenciatura & casada & $\begin{array}{l}\text { profesora de } \\
\text { secundaria }\end{array}$ & D.F. & programa de radio \\
\hline PM2 & 26 & licenciatura & casada & $\begin{array}{c}\text { profesora de } \\
\text { primaria }\end{array}$ & D.F. & libro y psicóloga \\
\hline PM3 & 46 & desconocida & casada & hogar & D.F. & desconocida \\
\hline PM4 & 30 & preparatoria & casada & hogar & D.F. & programa de radio \\
\hline PM5 & 41 & $\begin{array}{l}\text { carrera } \\
\text { técnica }\end{array}$ & casada & $\begin{array}{l}\text { empleada en } \\
\text { gobierno }\end{array}$ & $\begin{array}{l}\text { Estado de } \\
\text { México }\end{array}$ & programa de radio \\
\hline PM6 & 32 & licenciatura & casada & $\begin{array}{c}\text { profesora en } \\
\text { jardín de niños }\end{array}$ & D.F. & programa de radio \\
\hline PM7 & 43 & $\begin{array}{c}\text { primaria } \\
\text { inconclusa }\end{array}$ & casada & hogar & $\begin{array}{l}\text { Estado de } \\
\text { México }\end{array}$ & ya conocía la institución \\
\hline PM8 & 44 & secundaria & casada & hogar & D.F. & una conocida \\
\hline PM9 & 36 & $\begin{array}{c}\text { carrera } \\
\text { comercial }\end{array}$ & soltera & comerciante & D.F. & un amigo \\
\hline PM10 & 29 & licenciatura & soltera & trabajadora social & $\begin{array}{c}\text { Estado de } \\
\text { México }\end{array}$ & ya conocía la institución \\
\hline PM11 & 50 & $\begin{array}{c}\text { primaria } \\
\text { incompleta }\end{array}$ & viuda & comerciante & $\begin{array}{l}\text { Estado de } \\
\text { México }\end{array}$ & un conocido \\
\hline PM12 & 39 & $\begin{array}{l}\text { secundaria } \\
\text { incompleta }\end{array}$ & casada & hogar & D.F. & programa de radio \\
\hline PM13 & 44 & $\begin{array}{l}\text { pasante de } \\
\text { licenciatura }\end{array}$ & casada & $\begin{array}{l}\text { profesora de } \\
\text { bachillerato }\end{array}$ & $\begin{array}{c}\text { Estado de } \\
\text { México }\end{array}$ & programa de T.V. \\
\hline PM14 & 49 & licenciatura & soltera & $\begin{array}{c}\text { trabajadora } \\
\text { social }\end{array}$ & $\begin{array}{l}\text { Estado de } \\
\text { México }\end{array}$ & un amigo \\
\hline PM15 & 40 & primaria & casada & $\begin{array}{c}\text { trabaja } \\
\text { (no especifica) }\end{array}$ & $\begin{array}{c}\text { Estado de } \\
\text { México }\end{array}$ & su hija \\
\hline
\end{tabular}

\section{Cuadro 2 - Algunos datos de hombres que participaron en las sesiones} seleccionadas para el análisis

\begin{tabular}{|c|c|c|c|c|c|c|}
\hline \multicolumn{7}{|c|}{ VARONES } \\
\hline Paciente & Edad & Escolaridad & $\begin{array}{c}\text { Estado } \\
\text { civil }\end{array}$ & Ocupación & $\begin{array}{c}\text { Lugar de } \\
\text { residencia }\end{array}$ & Referencia \\
\hline PH1 & 35 & licenciatura & casado & arquitecto & D.F. & programa de radio \\
\hline PH2 & 44 & $\begin{array}{c}\text { licenciatura } \\
\text { incompleta }\end{array}$ & casado & desempleado & D.F. & terapeuta familiar \\
\hline PH3 & 47 & licenciatura & casado & profesor & $\begin{array}{c}\text { Estado de } \\
\text { México }\end{array}$ & programa de radio \\
\hline PH4 & 55 & licenciatura & casado & desempleado & D.F. & médico psiquiatra \\
\hline PH5 & 40 & $\begin{array}{c}\text { secundaria } \\
\text { incompleta }\end{array}$ & soltero & $\begin{array}{c}\text { vendedor de } \\
\text { perfumes }\end{array}$ & D.F. & anuncio en revista \\
\hline PH6 & 24 & $\begin{array}{c}\text { estudiante de } \\
\text { licenciatura }\end{array}$ & soltero & $\begin{array}{c}\text { promotor en } \\
\text { tienda }\end{array}$ & $\begin{array}{c}\text { Estado de } \\
\text { México }\end{array}$ & ya conocía la institución \\
\hline PH7 & 27 & secundaria & casado & comerciante & $\begin{array}{c}\text { Estado de } \\
\text { México }\end{array}$ & programa de radio \\
\hline PH8 & 52 & $\begin{array}{c}\text { sexto de } \\
\text { primaria }\end{array}$ & casado & fotomecánico & $\begin{array}{c}\text { Estado de } \\
\text { México }\end{array}$ & programa de T.V. \\
\hline PH9 & 51 & secundaria & divorciado & jubilado & $\begin{array}{c}\text { Estado de } \\
\text { México }\end{array}$ & ya conocía la institución \\
\hline PH10 & 36 & secundaria & casado & chofer de pipa & $\begin{array}{c}\text { Estado de } \\
\text { México }\end{array}$ & un conocido \\
\hline
\end{tabular}




\section{Los procedimientos}

La entrada al grupo fue paulatina y gradual. El primer contacto se realizó con las terapeutas del grupo a quienes se les expuso el proyecto en forma oral y escrita. Después se hizo presentación con el grupo a quienes se les explicó el objetivo del proyecto y se pidió su consentimiento para permitir las observaciones que en su mayoría, por petición de las terapeutas, se realizaron desde una cámara de Gesell. En seis ocasiones se compartió el espacio con el grupo debido a fallas técnicas en el audio o luz de la cámara y por petición de la observadora. A pesar del uso de la cámara de Gesell, el contacto con el grupo era habitual al inicio y término de las sesiones. Con frecuencia el grupo identificaba a la observadora como integrante del equipo terapéutico o estudiante de terapia sexual, no obstante los intentos por romper con dicha percepción. Pensamos que lo anterior era favorecido por los antecedentes académicos de la observadora - ser psicóloga y estudiar una maestría - y porque era más fácil entender la presencia de una persona ajena al grupo asociándola con lo que les era familiar.

Se registró en un diario lo acontecido en las sesiones, lo cual constituyó una primera sistematización y reducción del fenómeno que pretendíamos comprender, así como una primera interpretación de los datos. Dos diferentes tipos de registro coexistieron. El primero pertenece a un discurso indirecto a modo de relato, por parte de la observadora. El segundo registro evoca un discurso directo más parecido a la crónica, pues en él se intentó capturar los diálogos tratando de respetar pausas, palabras y turnos de habla. Durante el análisis, resultó de gran utilidad distinguir un discurso del otro porque eso nos permitió notar que el registro no fue uniforme, sino que constituyó un proceso.

\section{El malestar sexual y su relación con un modelo ideal de normalidad sexual}

El análisis de las crónicas mostró que en la terapia sexual los significados de malestar sexual se vinculan estrechamente con el establecimiento de un modelo ideal de normalidad sexual. Este modelo se refiere a un patrón de desempeño sexual al cual las personas debían adaptar sus prácticas sexuales para alcanzar los estándares de normalidad. Hemos denominado a ese modelo como ideal porque representa un patrón con el cual se compara y valora la experiencia individual. Como resultado 
del análisis de los materiales de campo, encontramos que el orden de género es un elemento constitutivo de dicho modelo en el sentido de que construye las diferencias entre los sexos y las define como naturales (SCOTT, 1996; BUTLER, 2000). Por ello a través del modelo se norman, reproducen y reafirman desigualdades y relaciones significantes de poder.

\section{En busca de una supuesta perfección del pene}

El modelo ideal de normalidad sexual tiene su fundamento en el patrón de respuesta sexual propuesto por Masters y Johnson, por lo cual el acontecimiento central es la penetración y en esencia la atención se dirige a los eventos y cambios ocurridos en los genitales. Así, el placer sexual es explicado fundamentalmente en términos biológicos, estableciéndose una fuerte conexión entre el patrón de respuesta sexual humana y la salud y normalidad sexual. De tal manera, se privilegia la existencia de una forma válida para experimentar placer sexual, cuyas divergencias son designadas como disfuncionales. ${ }^{2}$

En el mencionado modelo, la respuesta sexual de hombres y mujeres es presentada como complementaria, lo cual puede indicar una primacía implícita de la heterosexualidad genital. Por ello, la noción de placer sexual se desarrolla más acorde con construcciones androcéntricas de la sexualidad que con la experiencia cotidiana de las personas. Más aún, al estar definidas las diferencias entre los sexos por relaciones significantes de poder, la guía fundamental para el placer sexual de hombres y mujeres es el pene y la penetración vaginal. De esta forma, resulta fundamental la existencia de un supuesto funcionamiento correcto en donde la erección debe presentarse de manera paulatina, firme y constante, lo que garantiza que el pene siempre se encuentre listo para penetrar. Aún cuando Masters y Johnson (1976a) describieron cambios durante la vejez en la respuesta sexual, éstos fueron presentados como una pérdida en la capacidad normal de respuesta. Así, se estableció la creencia de que una persona sana y normal experimentaría la curva de respuesta sexual espontáneamente y sin dificultad alguna, y que tal acontecimiento garantizaría el acceso al placer.

Estas reflexiones se apoyan en numerosos diálogos registrados, donde el malestar sexual de los hombres se vincula estrechamente con los significados en torno al pene, y su relación con cierta construcción social de la masculinidad. Ser poseedor de un pene significaba para ellos la responsabilidad de proporcionar y obtener 
disfrute en la relación coital. Esto implicaba que debían ser expertos en el terreno erótico y controlar su órgano sexual sometiéndolo a una supuesta infalibilidad en cuanto a la capacidad de erección. A diferencia de las mujeres, los hombres no se cuestionaban sobre su capacidad de experimentar placer sexual, pues concebían que orgasmo y eyaculación eran sinónimos.

En este grupo, la demanda inicial de los hombres estuvo relacionada casi siempre con la preocupación por cubrir los estándares de perfección en el funcionamiento del pene. Con frecuencia ellos mostraron desconcierto ante su respuesta sexual y la percibían como anormal. Los principales motivos de consulta estaban relacionados con la falta de control en la eyaculación o en la erección. El siguiente fragmento ejemplifica lo anterior:

$\mathrm{PH6}^{3}$ : Vengo porque estoy preocupado por mi sexualidad. Yo nunca había tenido relaciones sexuales, sino hasta [hace] cuatro o cinco meses. Me ha ido mal, siento que no he llenado a mi pareja sexualmente, a veces tengo erección, pero ya sea que eyacule antes o....no sé, eso es lo que me pasa.

T2: ¿Qué creen que le pase a PH6?

PH3: Está asustado. Dice que se siente angustiado, yo creo que antes o después en esas cosas nos sentimos angustiados. La sapiencia popular dice que uno no debe fallar, a veces lo dicen como broma y a veces pasa, pero es cosa de aprender.

T2: Podríamos pensar que cuando uno aprende a manejar la torpeza se le desaparece más tarde. ¿Por qué lo debería hacer bien?, ¿por qué yo no debería asustarme? [...] Si se me para o no. (Se dirige a PHG) En la medida en que tú te angusties, la respuesta sexual no se da como se daría. La angustia tiene un efecto paralizador de cualquier actividad, como ya te ha sucedido automáticamente lo evitas. Cualquier actividad sexual no debe iniciarse en estado de angustia, incertidumbre. Por eso los ejercicios para trabajar la disfunción.

La presentación de PH6 denota un acercamiento previo al discurso sexológico, pues el uso de la palabra erección y su descripción de eyacular antes indica que existe para él una duración idónea de la erección, que podemos suponer proviene de la difusión del modelo ideal de normalidad sexual. El diálogo indica que PH6 y la terapeuta consideran que la respuesta sexual definida por ese modelo debe presentarse natural y espontáneamente. La sensación de malestar en este varón se relaciona con el desconcierto ante su propia respuesta sexual y el peso de la responsabilidad que asume en el placer sexual de su pareja. Al parecer, y tal como lo indica la participación de $\mathrm{PH} 3$, otros participantes comparten la sensación de malestar que producen las exigencias de la respuesta sexual masculina requerida por el modelo. Al nombrar la 
respuesta sexual de PH6 como torpe y producto de la angustia, la terapeuta parece asumir la existencia de un patrón único de respuesta sexual - que sería lo natural - y al cual se debe aspirar. La referencia a los ejercicios (tareas sexuales) y su liga con la palabra disfunción revela la lógica intrínseca de la terapia sexual, esto es, el objetivo es corregir la respuesta sexual considerada como anormal y adaptarla a los estándares del modelo. Así, al no cuestionarse sobre la preocupación de PH6 y delimitarla en los confines del modelo ideal de normalidad sexual, el enfoque sexológico minimiza o anula la complejidad del malestar sexual y afirma la construcción de un síntoma basado en una supuesta falla del paciente: el malestar proviene de la incertidumbre frente al modelo y no de un displacer nacido de la experiencia.

Las tareas indican la presencia de una contradicción no explicita en el discurso sexológico, pues al mismo tiempo que se plantea una supuesta naturalidad de respuesta sexual, se implementa un proceso de aprendizaje para adecuar un estándar fijado. Como hemos dicho, en el grupo que observamos las personas recibían un entrenamiento en sus prácticas sexuales por medio de la asignación de diversos ejercicios denominados tareas. Las actividades estaban encaminadas a que el o la paciente experimentara el orgasmo durante el coito heterosexual, lo cual implica designar al pene como el órgano responsable del placer sexual para ambos sexos y descubre una situación desigual de poder en la relación experto-paciente, pues el primero define por anticipado lo más conveniente para el segundo, restando importancia a la experiencia personal y, por tanto, al malestar.

La guía de tareas sexuales para hombres tiene como una de sus metas el control de la eyaculación durante la penetración. El siguiente fragmento retoma algunos de los puntos anteriores.

PH4: Hice la tarea. Tres stops, esa fue la última tarea. No he tenido relaciones desde hace un mes, por un curso que ella tiene, no he querido presionarla. Yo me he sentido muy bien.

T1: ¿Tú has intentado algo con ella sexualmente?

PH4: Pues sí, pero me da temor de que no funcione.

T1 lee una hoja escrita por PH4 en donde describe las sensaciones que tuvo durante el ejercicio, lo cual es parte de la tarea: ¿Por qué no te masturbas?

PH4: Sí lo hago, pero a la hora de dormir no. Temo no funcionar.

T1: Las sensaciones que describes son muy específicas, ve qué pasa a nivel del cuerpo, más general, ¿`como qué pasa?, ¿logras tener una erección? 
PH4: Sí, sí. Eyaculo la cuarta vez. Cuando estuve sólo en la casa pude hasta dos veces. La proximidad de la gente me afecta. La relación de pareja tambalea. La familia de mi esposa le afecta. Yo llevo sin trabajo seis meses.

T1: ¿¿Sientes que tu situación sexual se deterioró a partir de que no trabajas?

PH4: En abril no sólo eso, no funcioné sexualmente.

T1: ¿Quién buscaba la relación?

PH4: Casi siempre yo.

T1: Abre un poco la comunicación ¿cómo se siente ella? ¿qué necesitaría para sentirse atraída? vuelve a hacer el ejercicio, ubica a nivel de todo el cuerpo, a nivel general, percibe todo. ¿Por qué ella no te busca?, ¿qué pasa contigo a partir de que estás sin trabajo, con tu autoestima, salud, sueño?

Kaplan (1978b) nombra a la técnica mencionada en el testimonio "la maniobra de Semans" o "stop-start", y la aconseja como una técnica más adecuada que "el método del apretón” recomendado por Masters y Johnson (1976b). Esta técnica consiste en masturbarse y detener la actividad un poco antes de sentir la inminencia de la eyaculación, eso es nombrado como "stop". Una vez recuperado el control, el hombre debe volver a estimularse el pene para repetir la experiencia. El objetivo de tal actividad es entrenarlo para identificar las sensaciones corporales previas a la emisión del semen y, de esta forma, retardar la eyaculación. La pregunta que salta a la vista es ¿por qué es importante que los hombres aprendan a controlar el tiempo para eyacular?, pensamos que tal necesidad está vinculada con el supuesto de que el detonante del placer femenino es el pene erecto dentro de la vagina. Aunque no es nuestra intención generalizar un tipo de práctica sexual como la que produce o no placer sexual a las mujeres o los hombres, sí nos interesa revisar por qué un tipo específico de práctica sexual ha llegado a imponerse como modelo ideal. La idea de que la penetración vaginal es el punto culminante de las relaciones sexuales normales y saludables se fundamenta en una concepción esencialista de la sexualidad que la remite y delimita únicamente a fines reproductivos.

La creencia en un supuesto funcionamiento normal del pene establece que las variaciones serán consideradas como fracasos, disfunciones o anormalidades, lo cual producirá malestar en los sujetos que signifiquen de ese modo su desempeño sexual. Foucault (2000) plantea que lo calificado como patología o anormalidad por la ciencia sexual, tiene su origen en lo que la institución religiosa señalaba como pecado y atentado contra la voluntad divina expresada en la sexualidad 
"natural" reproductiva. De esta manera, aunque la ciencia pretendía separar a la sexualidad de su significación religiosa y pecaminosa, al mismo tiempo proporcionó nuevos parámetros para homogeneizar el deseo y la práctica sexual.

La respuesta sexual es múltiple y la idea de que existe un solo patrón de respuesta sexual incrementa la ansiedad de las personas que creen alejarse de los estándares de normalidad y salud. La universalización y descontextualización de la respuesta sexual ocasiona desconcierto cuando los hombres no entienden por qué "no funcionan bien". El discurso sexológico ha integrado en sus estudios factores de tipo emocional, etarios, situación del vínculo afectivo con la pareja, y otros, pero los retoma como elementos que alteran la "respuesta sexual normal". Si lo estadísticamente normal es lo que se presenta con mayor frecuencia estadística ¿por qué la normalidad no radica en la variabilidad de la respuesta sexual? Nos parece que una cuestión central es preguntarse por qué un tipo específico de respuesta sexual ha llegado a ser impuesto como la norma y defendido así por la ciencia sexual.

En el grupo de terapia sexual, nos percatamos de cierta ambigüedad con relación al papel central de la penetración. Al mismo tiempo que se decía expresamente que la penetración no era lo más importante en el acto sexual, la lista de tareas sexuales para varones y mujeres estaba basada en el modelo ideal de normalidad sexual, revelando así contradicciones de fondo. Un ejemplo es el siguiente diálogo:

PH4: Muy bueno mi fin sexual, lo logramos. Hubo erección alta, ella quedó satisfecha, seguimos jugando y luego otra vez. Yo ya no estaba preocupado como las otras veces.

T2: Ya aprendiste que en la vida lo lindo es todo, no sólo la penetración, las caricias. ¿Qué tal te fue con tu tarea?

PH4: Me dejaste ver[me] en el espejo y los cuatro arranques y tres stop. Lo hago todos los días.

T2: Vas a traer un ejercicio tres veces por escrito, quedamos que te toca cambio de tarea. Anota qué sensaciones son las que notas antes de eyacular. Un registro de tres veces y que te cambien la tarea.

Diversos autores (METCALF; HUMPHRIES, 1985; SEIDLER, 1995) han destacado que el desempeño eréctil es clave para afirmar la identidad sexual de los hombres, que el deseo sexual masculino se concibe como una fuerza irrefrenable y que la sexualidad masculina está indisolublemente ligada al pene y sus representaciones. Tras la revisión de distintos estudios en México, Szasz (1998) 
concluye que las caricias y expresiones eróticas sin penetración, por intensas que fueran, no eran consideradas relaciones sexuales por diversos grupos de hombres. La erección y penetración son dos eventos sobrecargados de significado y los genitales masculinos se pueden concebir separados del cuerpo como cobrando vida propia y representando valor, orgullo, prepotencia, fuerza y bienestar (CASTRO, 1998; GUTMANN, 2000). Dichos significados concuerdan con el modelo que encontramos y por eso remarcamos que diversas construcciones de género se entrelazan con esta sexualidad anclada en la reproducción. Así, el placer sexual se reduce a una mecánica sexual específica que intenta aislar una esencia verdadera y al hacerlo se diluye la posibilidad de entender la multiplicidad de la experiencia erótica humana. Esto es, al fragmentar su complejidad se incurre en simplificaciones excesivas que pueden llevar a concebir al placer sexual femenino como dependiente del pene y la penetración, deviniendo en la idea de que los hombres son seres sexuales activos, mientras que las mujeres no lo son. Un ejemplo lo constituye la equivalencia entre eyaculación y orgasmo en los hombres.

Diversas autoras han resaltado cómo al equiparar eyaculación y orgasmo, el placer masculino se presenta como un proceso fisiológico que implica una necesidad de desahogo sexual. Bajo esta premisa descansan ciertas construcciones de la sexualidad masculina, las cuales consideran que la excitación sexual está basada en impulsos biológicos dolorosamente intensos, que requieren un inmediato alivio (SZASZ, 1998). Vance (1989) ha advertido las implicaciones que tiene sobre el deseo y placer femenino el concebir a la sexualidad masculina en esos términos.

$\mathrm{Al}$ establecerse un tipo específico de respuesta sexual como normal y saludable, se buscan los factores que causan la desviación:

T2: ... ¿Cómo llegaste aquí?

PH5: Por medio de una visita al urólogo, él me recomendó, es mi estado psicológico y me mandaron para acá. El problema es seguir viviendo esa vida sexual, él me dijo que no es algo físico, sino las tensiones del trabajo, de acuerdo a las tensiones y presiones. Puedo dejar los problemas de la casa, pero no los del trabajo.

T2: O sea, ya no se te para.

PH5: Bueno, o sea, yo no duraba mucho tiempo, me costaba trabajo, luego sí me pasó eso... sí y eso es para mí importante, es el cincuenta por ciento de mi vida.

En la canalización de los pacientes a terapia sexual, los médicos jugaron un papel importante. Entre los especialistas existe la idea de que si la respuesta sexual 
no se adapta al modelo ideal se puede deber a factores de tipo físico o psicológico.

De manera particular, y dado que el modelo descansa sobre el supuesto funcionamiento correcto del pene, la sexualidad masculina se encuentra sujeta a vigilancia médica. Así, se han desarrollado un vocabulario, exámenes clínicos, medicamentos, métodos de diferenciación y criterios diagnósticos específicos para vigilar y regular médicamente la respuesta sexual de los hombres. Debido que ésta se concibe como cifrada en el cuerpo biológico, con frecuencia los hombres acudieron en primera instancia con el médico, quien realizó algunos exámenes clínicos o de laboratorio y cuando consideraba que los factores eran de índole no orgánico, referían al paciente a terapia sexual. Sin ignorar los factores orgánicos que median los procesos anatomofisiológicos de la respuesta sexual, el placer nunca podrá ser reducido a ellos. Hemos tratado de mostrar que esa conceptualización de la sexualidad ha sido planteada desde una postura que traspasa la comprensión de los procesos orgánicos del cuerpo y que ni la medicina, ni la terapia sexual la analizan o cuestionan, sino que la reproducen.

Al respecto comenta Tiefer (1995, p. 315):

La salud sexual se ha reducido en los hombres al funcionamiento eréctil del pene. La investigación y el tratamiento de la impotencia constituyen para los urólogos una subespecialidad médica nueva y muy brillante, y las tecnologías del diagnóstico y del tratamiento son una industria en pleno crecimiento. El campo de la sexualidad masculina se concentra en un órgano físico específico y dicta normas universalizadas de funcionamiento correcto e incorrecto. No existe un auténtico interés en la sexualidad de una persona, por no mencionar la de una pareja de una cultura y relación determinadas. Sólo hay normas universalizadas de órganos biológicos, como sucede con el corazón o los riñones.

Nos parece relevante señalar cómo es entendido el término "salud sexual" desde la perspectiva biomédica de la sexualidad. Tal y como señala Tiefer, cuando la sexualidad es explicada en términos de salud, la ciencia médica deposita al placer sexual en partes concretas del cuerpo y designa como saludable o no saludable su funcionamiento. Existen opiniones encontradas sobre la relación entre de los términos 'salud' y 'sexual', pues refieren que dicha unión puede ser significada ya sea como el acceso a ciertos derechos o como la base para legitimar ciertos criterios normativos de adaptación sexual. Creemos que la presente investigación proporciona elementos para repensar las implicaciones de asociar sexualidad con salud de manera acrítica. 


\section{Acerca del "verdadero" orgasmo}

Un evento que inquietaba particularmente a las mujeres del grupo que observamos y comandaba su decisión de acudir a terapia sexual era la preocupación por el orgasmo. De acuerdo al modelo ideal de normalidad sexual, el orgasmo es la parte culminante del placer sexual. En este contexto terapéutico y dado lo anteriormente expuesto, ese orgasmo sólo es verdadero bajo una forma específica, es decir, cuando es provocado por un pene durante la penetración vaginal. Algunas de las mujeres nunca habían experimentado un orgasmo, otras conocían la experiencia por medio de la masturbación y buscaban adaptarla al modelo normativo. También asistían mujeres que identificaban como primer motivo de consulta un "bajo deseo sexual" y en el transcurso de la terapia se interesaban por el orgasmo.

El orgasmo se describe en el DSM IV de la siguiente forma:

El ciclo completo de respuesta sexual puede dividirse en las siguientes fases: [1.Deseo 2.Excitación]

3. Orgasmo: Esta fase consiste en el punto culminante del placer sexual, con la eliminación de la tensión sexual y la contracción rítmica de los músculos del perineo y de los órganos reproductores. En el varón existe la sensación de inevitabilidad eyaculatoria, que va seguida de la emisión del semen. En la mujer se producen contracciones (no siempre experimentadas subjetivamente como tales) de la pared del tercio externo de la vagina. Tanto en el varón como en la mujer el esfínter anal se contrae de manera rítmica. (DSM-IV, 1995, p. 506, paréntesis del texto original.).

La definición anterior presenta varias dificultades. Al señalar al orgasmo como el "punto culminante" del ciclo de respuesta sexual, determina que no hay relación sexual completa sin orgasmo. Al basar la definición en los cambios anatomofisiológicos acontecidos en los órganos genitales, relaciona estrechamente a la sexualidad con la fisiología reproductiva. Así, en un intento de unificar criterios y sustentarlos en evidencias objetivas, este enfoque reduce y desconoce la infinita posibilidad del erotismo humano. Otro aspecto que concierne directamente al género es que la definición separa y especifica el orgasmo por sexo, pero en ambos casos describe la experiencia refiriéndola a los órganos que participan en la penetración vaginal. En los varones, el orgasmo queda equiparado a la eyaculación, evento observable. En la mujer, es de resaltar el hecho de que las contracciones vaginales pueden pasar desapercibidas para las propias mujeres, lo cual sugiere una cierta desestimación de la experiencia erótica. 
Algunas preguntas de las mujeres que solicitaban atención eran: ¿cómo son los verdaderos orgasmos?, ¿qué es un orgasmo?, ¿lo que siento es un orgasmo?, ¿por qué no tengo orgasmo en la relación sexual? Con frecuencia les preocupaba obtener un orgasmo producido por el pene de su compañero y les era difícil asimilar que el pene no fuera el "detonante" del mismo:

PM10: Yo estoy aquí porque siempre me inquietó. Siempre me llamó mucho la atención, ahora que conocí a una pareja que era su primera vez, me parece que es una oportunidad para aprender una sexualidad completa. Existe la idea de aprender, como cuando dicen que los hombres tienen que saber de mecánica y las mujeres a cocinar.

T1: ¿Cómo te sientes con tu sexualidad?

PM10: Me siento bien, puede ser plena. Me gusta aprender, siempre he creído que esta es una de las mejores oportunidades, no sólo me gusta aprender teoría o investigación. Que los dos nos conozcamos.

T1: ¿Tú tienes orgasmos?

PM10: Esa es otra cosa, yo no sé si lo que yo siento es un orgasmo, a lo mejor sí lo es y no lo sé, otra cosa que quiero saber es si yo tengo orgasmos o no. Es una duda muy grande. Me puse a investigar en los libros o el internet qué es un orgasmo, y no lo sé, nunca dicen claro.

T1: ¿Tú te has masturbado?

PM10: No, no, casi no, digamos que soy novata, no, nunca, sólo cuando hay un roce, me baño, pero no, nunca.

Alguien del grupo que no veo: Dicen que cuando tengas un orgasmo nunca vas a tener duda.

T2: De vez en cuando sí aparecen las dudas, porque en las revistas los orgasmos son de siete kilómetros o con luces.

En el grupo: Sí, sí, es cierto. (risas).

Este discurso médico-sexológico apoya la idea de que físicamente las mujeres pueden tener un orgasmo y no experimentarlo. En el fragmento anterior, la terapeuta hace referencia a cómo son presentados los orgasmos en las revistas y el grupo confirma la veracidad de su frase. Recordemos cómo, en efecto, en los medios masivos de comunicación se representa mitificada a la experiencia del orgasmo femenino. Muchas personas hacen de esa imagen la norma de lo que debe ser y comparan su experiencia con ella; mientras mayor distancia exista entre ambas, aumentará la posibilidad de significar con malestar la vida sexual, 
porque se tiene la convicción de que lo representado por los medios es la imagen fidedigna de un "verdadero" orgasmo.

Nos parece relevante resaltar las construcciones que nuestra cultura ofrece en torno a la importancia del orgasmo, evento que seguramente en otras culturas tiene un significado y estatus diferente. El orgasmo es inaprensible, un evento singular.

PM11: Me toco la vagina y siento cosquillitas, como que está dormida...//... No sé claramente lo que es un orgasmo.

PM13: Eso bonito que dices que sientes, con tus palabras.

T2: Es un movimiento que sube y que sube y guau hace una expansión.

Alguien que no veo. En mi caso es una especie de tensión, como adormecimiento. La forma en que he sentido más placer es por atrás, no sé si sea la posición, y luego sintiendo algo tibio. Yo sí me lubrico, pero no siempre, cuando me siento seca usamos gel porque si no me lastimo.

Aquí las mujeres intentan describir la experiencia sensorial del orgasmo usando sus palabras. La preocupación por saber cómo es el orgasmo y si lo sienten o no, tiene que ver con cómo ha sido construida dicha experiencia desde el modelo ideal de normalidad sexual. El discurso científico señala a las contracciones vaginales como el referente material más claro del orgasmo femenino por lo que ellas esperaban atentas sensaciones extraordinarias en la vagina e insistían con frecuencia que debía haber algo que denominaban "los verdaderos orgasmos":

Están en un subgrupo PM7, PM4, PM9 y T1. T1 sale al baño les pide que le vayan explicando la tarea a PM9, quien asiste por primera vez. Ellas le indican que el primer paso es verse desnuda frente al espejo y le dicen cómo hacerlo. Cuando terminan PM9 les pregunta.

PM9: y ¿ya tuvieron orgasmo?

Risas

PM7: Sí, bueno... no sé como serán los verdaderos orgasmos, pero yo sí siento algo.

Entra T1 y les pregunta sobre qué hablan. Ellas contestan que discuten acerca de cómo serán los verdaderos orgasmos.

T1: ¿Y a poco todas vamos al baño iguales?, cada quien lo vamos a sentir distinto, una misma lo siente de diferentes formas. Continúa hablando sobre la tarea.

No es nueva la reflexión sobre los "verdaderos" orgasmos de las mujeres. Ya en 1968, Anne Koedt publicó su artículo El mito del orgasmo vaginal. En él planteó 
cómo el orgasmo vaginal alcanzado exclusivamente mediante el coito había sido durante mucho tiempo para los expertos una nota clave sobre la normalidad femenina y la salud sexual de las mujeres. Al revisar los planteamientos de Koedt, Gerhard (2001) expone que los estudios sexológicos como los de Kinsey y Masters y Johnson proporcionan argumentos para considerar que la sexualidad masculina y femenina tenían más similitudes que diferencias. Para la autora, los textos sexológicos contaban incluso con el potencial para alterar sustancialmente no sólo la práctica heterosexual, sino también la asociación profundamente arraigada entre la sexualidad de una mujer y su identidad de género. Ella relata cómo las feministas de la segunda ola (1968-1973) cuestionaron el carácter innato de la heterosexualidad y defendieron la idea de una sexualidad femenina autónoma del coito con un hombre, apoyándose en las investigaciones sexológicas.

Sin embargo, en el ejemplo del contexto que aquí detallamos el resultado de la intervención terapéutica parece ser lejano a una experiencia "liberadora" en el sentido de que, en lugar de favorecer la reflexión sobre las condiciones de sujetación que hacen difícil el ejercicio del placer, se ofrece un modelo normativo para que los sujetos realicen prácticas de sí tendientes a la normalización. Resulta alentador pensar una sexualidad construida sobre bases más equitativas; sin embargo, es necesario enfatizar que, aun cuando los textos sexológicos contengan elementos para pensar en dichos términos la sexualidad, los significados reportados en numerosos estudios revelan que existen contextos en los que la interpretación es diferente.

Los orgasmos que no se adecuan al modelo ideal de normalidad sexual, aun cuando se disfrutan, no parecen recibir el mismo valor. En el siguiente fragmento una mujer habla de cómo aprendió a sentir orgasmos.

PM3 habla de que agradece a una persona que le enseñó a masturbarse a los diecisiete años; esa mujer mayor le decía que si se frotaba entre las piernas iba a sentir muy rico, PM3 dice que lo hacía con un trapo y sentía orgasmo, pero que lo dejó porque pensaba que era malo. Después lo retomó, aún de casada, con el esposo no se siente excitada, se masturba, y entonces se siente culpable. PM5 pregunta si se masturba delante del esposo y PM3 le contesta que no porque siente que él se va a ofender si ella se pone el trapo en vez del pene (asi se masturba, con un trapo entre las piernas), ella no le ha contado del trapito, ni de cómo se masturba. PMG le dice que debería permitirse hacer eso con él. PM5 dice que ella sí introduce su vibrador en la relación, PH1 también opina que ella debería introducir el trapito en su relación. 
El malestar de PM3 se asocia con la construcción de una forma válida para experimentar el placer sexual establecida en el modelo. Es evidente que la construcción acerca de la primacía del pene como detonante del placer sexual femenino obstaculiza la validación de la propia experiencia para esta mujer. La anterior descripción de la técnica de masturbación fue quizá la más explicita que encontramos en el transcurso de las observaciones, aún cuando por la rapidez con que PM3 narró su historia fue necesario usar un discurso indirecto para capturarla. Es oportuno señalar que aunque la técnica de masturbación en el caso de los hombres es explícita y forma parte de las tareas sexuales, en el caso de las mujeres no hay indicaciones específicas o técnicas, únicamente se indica tocar los genitales y tener sensaciones o autoestimularse con lubricante hasta el orgasmo. Se puede decir que debido al papel prioritario que se le asigna al pene en el placer femenino, éste no se puede pensar sino en función de aquél, y por lo tanto toda técnica de autoestimulación es meramente preparatoria. Así, a partir de esa perspectiva, el camino al orgasmo femenino queda sujeto al desempeño del pene y oculto tras la importancia asignada a la penetración.

$\mathrm{Al}$ igual que en los hombres, en diversas ocasiones la falta de adecuación al modelo era percibida por las mujeres como una "falla" en el funcionamiento del cuerpo. Esto se relaciona con el discurso que el enfoque biomédico tiene en torno al malestar sexual.

PM12 Llora y continúa hablando de dificultades con su familia. Cambia el tema y dice: comencé a preocuparme si es algo de mi organismo, de adentro. Yo quiero tener logros pronto ¿y si no es nada más y pueda ser algo físico, el que yo no tenga orgasmos? Antes de mi menstruación, unos días antes, estoy que no quiero ni que me vean...//...para mí el sexo no es primordial, yo puedo vivir sin el sexo.

La preocupación sobre un supuesto origen orgánico del síntoma remite a cómo ha sido construido el modelo ideal de normalidad sexual. La ausencia de deseo se encuentra patologizada y la paciente intenta explicar su supuesta patología con el proceso hormonal de la menstruación. La medicina occidental ha tratado de solucionar los problemas del organismo con sustancias y la sexualidad no ha escapado a ello, como demuestra la gran difusión de medicamentos que pretenden incidir en el funcionamiento eréctil de los genitales masculinos. El fragmento que sigue aborda la presentación de una sustancia para las mujeres. 
T1: A mí me sugirieron este producto. Saca una caja con una especie de tubitos. Se

llama viacrime y es como viagra para las mujeres. Me costó dos mil pesos, si de repente alguna quiere probar qué se siente [...] Las puedo vender sueltas en doscientos pesos. Le preguntan qué se siente, si ella ya probó [...] sí, se sienten cosas, se siente mayor sensibilidad, es más fácil alcanzar el orgasmo, no que la cremita y ya está el orgasmo, aquí dice que son tres o cuatro veces de uso. Yo me puse la mitad. Bueno las traje para que vean que ahí está esto. No es la panacea, no es la solución, pero sí ayuda, esto ayuda relajando, llenando de sangre los labios, sí ayuda.

T2 lee un folleto que acompaña al producto, está en francés y auxilia en la traducción, una frase dice: su deseo de hacer el amor aumenta y aumenta...

PM5: ¡Ah!, está pensado en los señores.

Risas en el grupo.

T2 continúa la traducción: es una crema que promueve la sensibilidad en el clítoris...

PM5: Vamos a hacer tandas

T1: Bueno, que sepan que aquí está. Si se ingiere, no pasa nada, se puede sexo oral.

PM3: Va a salir carito.

T2: Así que mejor a trabajar con sus chambas.

Risas. Se dividen en subgrupos

La vigilancia de la sexualidad desde el campo médico ha desencadenado un progresivo interés en la industria farmacéutica formal para desarrollar sustancias que logren adecuar la respuesta sexual a los estándares de normalidad. Esto se ha dirigido con mucha mayor fuerza y constancia a los hombres, a quienes una industria creciente a gran escala les promociona válvulas, prótesis genitales y pastillas, entre otros productos. Quizá la dificultad de apresar el orgasmo femenino había salvado a las mujeres de la regulación farmacológica del placer, pero aquí vemos cómo también se comienza a filtrar la industria del sexo en ese campo. Los laboratorios pueden así lucrar con el malestar generado por el modelo, al ofrecer la ilusión de que el fármaco sustituye el deseo, la imaginación o la sensibilidad.

\section{Conclusiones}

Las disfunciones sexuales y su tratamiento mediante la terapia sexual sólo pueden ser avalados por una interpretación biomédica de la sexualidad. Tal enfoque postula la existencia de una supuesta sexualidad natural que puede ser aislada de los sujetos 
y manipulada en nombre de una concepción particular de la salud y el bienestar. Los significados encontrados en este estudio apoyan la idea de que la experiencia sexual trasciende los límites del modelo ideal de normalidad sexual que sostiene el tratamiento sexológico. Colocarse en el lugar de experto y poseer la verdad sobre el sexo implica ignorar que nadie es inmune a los efectos y el ejercicio del poder y que ni la ciencia, ni las buenas intenciones nos libran de dicho ejercicio.

Hemos revisado que la clasificación de los eventos sexuales en categorías dicotómicas es una práctica cuya historia revela un complejo entramado de circunstancias políticas y sociales que trascienden a la biología. Aunque bajo el orden de género siempre interpretamos el cuerpo como sexuado, el placer no puede ser reducido a sus traducciones. Es indispensable entender que la investigación sobre sexualidad requiere de la problematización y el análisis de las nociones y categorías establecidas como verdaderas por la ciencia sexual. Aún cuando la presente investigación tiene como escenario de estudio un grupo específico de terapia sexual, el discurso desarrollado ahí en torno al malestar sexual refleja diversas construcciones sobre la sexualidad que trascienden dicho espacio.

\section{Referencias}

ALCÁNTARA, E.; AMUCHÁSTEGUI, A. Terapia sexual y normalización: significados del malestar sexual en mujeres y hombres diagnosticados con disfunción sexual. Rev. Tramas, México, n. 22, p. 195-221, 2004.

- Terapia sexual y normalización: significados del malestar sexual en mujeres y hombres diagnosticados con disfunción sexual. Revista de Estudios de Género La Ventana, México, v. II, n. 20, p. 157-194, 2004.

ÁLVAREZ-GAYOU, J. Sexoterapia integral. México: Manual Moderno, 1986. 428p.

ASOCIACIÓN AMERICANA DE PSIQUIATRÍA. Manual diagnóstico y estadístico de los trastornos mentales DSM-IV. México: Masson, 1995.

BUTLER, J. El género en disputa. El feminismo y la subversión de la identidad. México: Paidós, 2000. 193 p.

CASTRO, R. Uno de hombre con la mujer es como una corriente eléctrica: subjetividad y sexualidad entre los hombres de Morelos. Rev. Debate feminista, México, v. 18, p. 105-130, 1998.

FOUCAULT, M. Historia de la sexualidad, v. I. México: Siglo XXI, 2000. 194 p.

GERHARD, J. De vuelta a "El mito del orgasmo vaginal": el orgasmo femenino en el pensamiento sexual estadounidense y el feminismo de la segunda ola. Rev. Debate Feminista, v. 23, p. 220-253, 2001. 
GONEMAN, C. Nymphomania: the historical construction of female sexuality. SIGNS, invierno, p. 337-367, 1994.

GUTMANN, M. Ser hombre de verdad en la Ciudad de México, México: El Colegio de México, 2000.

JACKSON, M. 'Facts of life' or the erotizacion of women's oppression? Sexology and the social construction of heterosexuality. In: CAPLAN, P. (ed.). The cultural construction of sexuality. London: Routledge, 1987. p. 54-81.

KAPLAN, H. La nueva terapia sexual, v. I y II. Madrid: Alianza, 1978a. . Manual ilustrado de terapia sexual. México: Grijalbo, 1978b. . Trastornos del deseo sexual. Barcelona: Grijalbo, 1985.

KOEDT, A. El mito del orgasmo vaginal. Rev. Debate Feminista, México, v. 23, p. 254-263, 2001. MASTERS, W.; JOHNSON V. Incompatibilidad sexual humana. Buenos Aires: Intermédica, 1976b. . Respuesta sexual humana. Buenos Aires: Intermédica, 1976a.

METCALF, A.; HUMPHRIES, M. The sexuality of men. London: Pluto Press, 1985.

ORGANIZACIÓN MUNDIAL DE LA SALUD. Clasificación internacional de Enfermedades. CIE10. Madrid: Meditor, 1995.

SCOTT, J. El género: una categoría útil para el análisis histórico. In: LAMAS, M. (comp.). El género: la construcción cultural de la diferencia sexual. México: Porrua y PUEG-UNAM, 1996. p. 265-302.

SEIDLER, V. Los hombres heterosexuales y su vida emocional. Rev. Debate Feminista, v. 2, p. 78$111,1995$.

STRAUSS, A.; CORBIN, J. Basics of qualitative research: grounded theory procedures and techniques. Ney York: Sage, 1990. 270 p.

SZASZ, I. Los hombres y la sexualidad: aportes de la perspectiva feminista y primeros acercamientos a su estudio en México. In: LERNER, S. (ed.). Varones, sexualidad y reproducción. México: El Colegio de México, 1998. p. 137-162.

TIEFER, L. El sexo no es un acto naturaly otros ensayos. Madrid: Talasa, 1995.361 p.

VANCE, C. Placer y peligro. Explorando la sexualidad femenina. Madrid: Talasa, 1989. 228 p.

WEEKS, J. El malestar de la sexualidad. Significados, mitos y sexualidades modernas. Madrid: Talasa, $1993.426 \mathrm{p}$. Sexualidad. México: Paidós, 2000. 131 p. 


\section{Notas}

* Una versión similar de este artículo fue publicada en la Revista de Estudios de Género La Ventana de la Universidad de Guadalajara, y Revista Tramas de la Universidad Autónoma Metropolitana-Xochimilco. Las referencias bibliográficas completas de estos artículos se encuentran en la bibliografía del presente trabajo. Agradecemos a los editores su consentimiento para la publicación del artículo en la Revista Physis.

${ }^{1}$ No contamos con el permiso de las terapeutas para reproducir las listas de tareas, sin embargo más adelante discutiremos acerca de algunas de las tareas sexuales y sus implicaciones.

2 Aunque en ocasiones los sexólogos asocian la disfunción eréctil con la angustia producida por la exigencia de desempeño sexual asociada con cierta definición de la masculinidad, el tratamiento se dirige más a la supresión del síntoma (a través de ejercicios o incluso del uso de fármacos) que a la crítica del orden de género que sustentaría esa supuesta afección.

${ }^{3}$ Con el fin de respetar la confidencialidad, se asignaron claves a los registros. Se utilizó la letra "T" para terapeuta, "P" para paciente, "M" para mujer y "H" para hombre, así como números consecutivos para distinguir a los participantes. Por ejemplo, la clave PH6 de este ejemplo designa a un paciente hombre determinado. 
Sexual therapy and normalization: meanings of sexual discontent in women and men diagnosed with sexual dysfunction

In the last 25 years, professionals and institutions who are interested in spreading sexual therapy have become firmly established in urban areas of Mexico. The treatment of sexual dysfunctions includes both pharmaceutical treatment and sexual training. Sociohistorical studies have indicated the particularities and limitations of biomedical approaches to sexual difficulties. In this paper, we present some reflections around the concept of sexual dysfunction and the biobehavioral procedures that support their treatment in sexual therapy. This paper intends to understand the meanings of sexual discontent in a group of sexual therapy that took place in Mexico City. The group, defined as open and mixed, had a two-hour session once a week. Although we carried out non-participant observation for a one year period, we selected 20 sessions for a final analysis. Three sexual therapists, 15 female and 10 male patients participated in the sessions selected for the analysis. Qualitative analysis of dialogues carried out in sexual therapy group demonstrated that in sexual therapy the meanings of sexual discontent are closely linked to an ideal model of sexual normality. This model refers to a standard of sexual performance to which people should adapt in order to comply with normality standards. Gender is constitutive of said model supported by sexual therapy, and thus inequalities and significant power relations are regulated, reproduced and reaffirmed through it.

> Key words: heterosexuality, sexual therapy, sexual dysfunction, sexology. 